COLABORACIÓN ESPECIAL

\title{
ORGANISMOS MODIFICADOS GENÉTICAMENTE: UNA NUEVA AMENAZA PARA LA SEGURIDAD ALIMENTARIA
}

\section{Liliane Spendeler}

Amigos de la Tierra España - Miembro de Amigos de la Tierra Internacional

\section{RESUMEN}

Este artículo analiza todos los aspectos referentes a la seguridad alimentaria relacionados con la introducción de los organismos modificados genéticamente en la agricultura y la alimentación. Se discuten las incertidumbres asociadas a la inserción de genes extraños en organismos, facilitando ejemplos de efectos imprevistos e indeseados y de inestabilidades de los organismos así fabricados artificialmente. Luego se aportan datos tanto de agencias oficiales como de la literatura existente, que cuestionan la seriedad y fiabilidad de los análisis de riesgo sobre la inocuidad para la salud de estos organismos y se discute la falta casi absoluta de estudios científicos que analicen la seguridad/peligrosidad de los alimentos transgénicos para la salud. Dadas todas estas incógnitas, se tienen que tomar en cuenta otros factores, en particular la contaminación genética de los cultivos no modificados genéticamente, que empieza a ser generalizada en algunas partes del mundo. No poder dar marcha atrás en caso de problemas resulta irresponsable. Otros elementos importantes son los impactos sobre el medio ambiente (como la aparición de resistencias en insectos, la pérdida de biodiversidad, el aumento de los productos químicos empleados) con repercusiones indirectas sobre la salud y/o la futura producción de alimentos. Por último se introducen elementos de discusión sobre la seguridad alimentaria en términos de disponibilidad de alimentos y soberanía alimentaria, dado que el mercado de las semillas transgénicas y los agroquímicos asociados está copado por cinco grandes empresas transnacionales. La conclusión hace un análisis de la contribución de la agricultura biotecnológica a la sostenibilidad.

Palabras clave: Organismos modificados genéticamente. Alimentos modificados geneticamente. Plantas modificadas genéticamente. Alimentos transgénicos. Seguridad alimentaria. Etiquetado de alimentos.

\section{ABSTRACT \\ Genetically Modified Organisms: A New Threat to Food Safety}

This article analyzes all of the food safety-related aspects related to the use of genetically modified organisms into agriculture and food. A discussion is provided as to the uncertainties related to the insertion of foreign genes into organisms, providing examples of unforeseen, undesirable effects and of instabilities of the organisms thus artificially fabricated. Data is then provided from both official agencies as well as existing literature questioning the accuracy and reliability of the risk analyses as to these organisms being harmless to health and discusses the almost total lack of scientific studies analyzing the health safety/dangerousness of transgenic foods. Given all these unknowns, other factors must be taken into account, particularly genetic contamination of the non-genetically modified crops, which is now starting to become widespread in some parts of the world. Not being able of reversing the situation in the even of problems is irresponsible. Other major aspects are the impacts on the environment (such as insects building up resistances, the loss of biodiversity, the increase in chemical products employed) with indirect repercussions on health and/or future food production. Lastly, thoughts for discussion are added concerning food safety in terms of food availability and food sovereignty, given that the transgenic seed and related agrochemicals market is currently cornered by five large-scale transnational companies. The conclusion entails an analysis of biotechnological agriculture's contribution to sustainability.

Key words: Genetically modified organism. Genetically modified food. Genetically modified crops. Food safety. Food labelling.

Correspondencia:

Liliane Spendeler

Amigos de la Tierra España

Avda. de San Fermin, 34

28041 Madrid

Correo electrónico: transgenicos@tierra.org 


\section{INTRODUCCIÓN}

Los organismos modificados genéticamente han hecho su aparición en la agricultura comercial hace diez años. Desde entonces la superficie sembrada con cultivos transgénicos ha ido aumentando progresivamente hasta alcanzar 67,7 millones de hectáreas en todo el mundo en 2003, según datos de la industria biotecnológica no corroborados por fuentes independientes ${ }^{1}$. Sin embargo, según la misma fuente, el número de países que siembra semillas modificadas genéticamente es muy reducido, ya que cerca del 99\% de la producción transgénica se realiza en 5 países y solamente otros 13 tienen alguna experiencia con cultivos transgénicos. Los cultivos con modificación genética sembrados actualmente a gran escala son la soja, el maíz, el algodón y la colza. Dos propiedades los caracterizan: la tolerancia a un herbicida y/o la producción por la planta de una toxina para el control de las plagas (plantas $\mathrm{Bt})$.

Paralelamente al crecimiento de la superficie de cultivos transgénicos en algunos países hemos asistido a un progresivo aumento del rechazo global hacía los cultivos y alimentos transgénicos. La oposición apareció en todos los continentes: la Unión Europea adoptó una moratoria a la siembra comercial y desarrolló una legislación restrictiva (aunque todavía incompleta), se impusieron prohibiciones en países asiáticos y latinoamericanos y muchos países del Sur rechazaron la ayuda alimentaria transgénica. En general, los consumidores de todo el mundo se han mostrado reticentes a adoptar los alimentos transgénicos.

Este rechazo está justificado por el hecho de que las empresas biotecnológicas fracasaron en comercializar productos que tuvieran beneficios claros para los consumidores y los agricultores, a la vez que la introducción en la agricultura de semillas transgénicas creó nuevos problemas alarmantes, entre ellos la contaminación genética. Por otra parte, los organismos modificados genéticamente han sido introducidos sin que hubiera una comprensión adecuada de sus impactos ambientales, socio-económicos y sanitarios. Los casos de contaminación con cultivos transgénicos ilegales (como los escándalos del 'StarLink' o de los 'biofarmacéuticos' en Estados Unidos y la contaminación del maíz mexicano) demuestran que se sabe muy poco sobre los impactos y las consecuencias de la liberalización al medio ambiente de los cultivos transgénicos.

En este contexto es legítimo que la sociedad reflexione y opine sobre la conveniencia de utilizar organismos modificados genéticamente en la agricultura y en la alimentación. Este artículo aporta elementos para una reflexión sobre el tema concreto de la seguridad alimentaria, claramente relevante en el debate sobre la introducción de los organismos modificados genéticamente en la producción de alimentos.

\section{INCERTIDUMBRES SOBRE LA INGENIERÍA GENÉTICA}

La obtención de organismos modificados genéticamente, que consiste en incorporar genes de otras especies a su ADN, se fundamenta en un modelo determinista que considera la acción de los genes lineal e independiente de otros factores internos o externos en la determinación de los caracteres. Pero los recientes descubrimientos demuestran que la realidad es mucho más compleja y que queda mucho por descubrir antes de tener una comprensión profunda del funcionamiento genético de los seres vivos ${ }^{2-4}$. Por ejemplo las interconexiones entre genes o la influencia del ambiente en el que vive el organismo parecen fundamentales en la expresión de los genes. Por lo tanto con el nivel de conocimiento actual resulta imposible prever todos los efectos de la inserción de genes extraños en el ADN de un organismo. De ahí la alta probabilidad de aparición de efectos imprevistos e indeseados así 
como de inestabilidades genéticas en los organismos modificados genéticamente. Ya son conocidos algunos casos de ambos efectos. Por ejemplo, en los últimos años se han detectado incertidumbres o errores en la descripción por las empresas de los genomas de la soja Roundup Ready ${ }^{5}$, del maíz NK603 (ambos de Monsanto) y del maíz Bt11 de Syngenta $^{7}$ (todos ellos aprobados para entrar en la cadena alimentaria). Por otro lado están apareciendo pruebas de que la mayoría de los eventos transgénicos aprobados en la Unión Europea presentan inestabilidades, como el caso del maíz Bt176 de Syngenta, del maíz MON810 de Monsanto (estos dos se cultivan en España) y del maíz Bt25 de Bayer CropScience ${ }^{8}$.

Estas inestabilidades y la incapacidad de predecir las consecuencias de la inserción de genes en organismos extraños se hace aun más peligrosa cuando éstos se liberan al medio ambiente y se integran en ecosistemas complejos, donde indudablemente reaccionarán y evolucionarán en función de las condiciones externas y donde tendrán oportunidad de interaccionar con el resto de los seres vivos presentes.

La ingeniería genética, tecnología radicalmente nueva y capaz de producir seres vivos que nunca se hubieran obtenido mediante mecanismos naturales, introduce un factor importante de inseguridad alimentaria por la imprevisibilidad, inestabilidad y, como consecuencia, la incertidumbre que le son inherentes.

\section{¿SON LOS ALIMENTOS TRANSGÉNICOS LOS MÁS SEGUROS DEL MERCADO?}

Partiendo de la base de que los organismos modificados genéticamente son seres totalmente nuevos y que ningún equivalente ha entrado nunca en la cadena alimentaria, y tomando en cuenta las grandes incógnitas que les caracterizan, parece legítima la exigencia de un análisis exhaustivo antes de que entren en la alimentación de los ciudadanos, así como un posterior seguimiento y control.

La primera observación en relación con este asunto es que la falta de estudios experimentales independientes sobre la inocuidad o la peligrosidad de los alimentos transgénicos para la salud humana es evidente. Varias revisiones bibliográficas concluyen que las investigaciones científicas disponibles para poder comer alimentos transgénicos sin temor son insuficientes ${ }^{9,10}$.

Además de la escasez de investigación en general los análisis de riesgo llevados a cabo caso por caso antes de las aprobaciones de eventos transgénicos a nivel europeo dejan mucho que desear. En primer lugar estos análisis se basan en las informaciones y experimentos realizados por las propias empresas, estudios en general incompletos y que plantean serias dudas sobre su objetividad. Por regla general, no se verifican con estudios independientes los datos aportados, ni las autoridades competentes europeas piden toda la información complementaria que cabría esperar sobre aspectos dudosos de los expedientes. Por lo tanto, a menudo importantes aspectos concretos no se evalúan adecuadamente. Por ejemplo los posibles efectos alergénicos del maíz MON863 de Monsanto o del maíz Bt11 de Syngenta, las alteraciones en el metabolismo del arroz LLRICE62 de Bayer CropScience, las diferencias de composición entre la colza GT73 de Monsanto y su equivalente no transgénico o los posibles efectos cancerigenos del maíz NK603 de Monsanto no han sido evaluados correctamente en los expedientes de las empresas ${ }^{11}$ y en cuatro de estos casos la autoridad competente europea, European Food Safety Authority (EFSA), ya ha dado su visto bueno a la aprobación, pasando por alto estas deficiencias.

De hecho, en varias ocasiones agencias oficiales de países europeos han expresado una opinión desfavorable a la aprobación de determinados eventos transgénicos o han 
subrayado fallos en la evaluación de riesgos. Fue el caso por ejemplo del maíz Bt11 de Syngenta, aprobado el 5 de mayo de 2004 unilateralmente por la Comisión Europea sin el respaldo de los Estados Miembros: en noviembre de 2003, la Agencia Francesa de Seguridad Sanitaria de los Alimentos revisó el dossier y concluyó que sobre la base de la información facilitada por Syngenta «no se pueden descartar efectos imprevistos» y que eran necesarios más estudios antes de llegar a conclusiones sobre la seguridad del maíz para la salud humana ${ }^{12}$; las autoridades belgas cuestionaron la calidad de la investigación de Syngenta para identificar si los genes habían sido insertados como se esperaba ${ }^{13}$; un informe del gobierno austríaco concluyó que ningún estudio sobre toxicidad había sido llevado a cabo con la planta entera, que no existían pruebas sobre los efectos a largo plazo de la nueva proteína y que los estudios sobre las reacciones alérgicas eran insuficientes ${ }^{14}$. El caso del maíz Bt176 de Syngenta proporciona otro buen ejemplo. La Comisión Europea dio luz verde a este evento en diciembre de 1996, a pesar del voto negativo de 13 de los 15 Estados Miembros. Posteriormente, Austria, Luxemburgo e Italia prohibieron este tipo de maíz en su territorio tanto para cultivo como para importación, siendo respaldados más tarde por Dinamarca y Suecia, y luego por Alemania. Los argumentos de estos países en contra del maíz Bt176 se referían tanto a preocupaciones sanitarias (transferencia del gen de resistencia a ampicilina a bacterias patógenas) como ambientales (efectos de la toxina Bt sobre especies no objetivo y la biodiversidad, aparición de resistencia al Bt en insectos y persistencia de la toxina en el suelo). Hubo que esperar hasta el año 2004 para que las autoridades europeas se dieran cuenta que efectivamente el maíz Bt 176 podría presentar un riesgo para la salud humana y prohibieron su empleo comercial a partir del 1 de enero de $2005^{15}$. Mientras tanto en España lo hemos estado comiendo durante siete años.

De manera más general, organismos oficiales de diferentes Estados Miembros de la Unión Europea han expresado a lo largo de los últimos años su preocupación en cuanto a la evaluación de los riesgos sanitarios de los organismos modificados genéticamente. Así, la Agencia Francesa de Seguridad Sanitaria de los Alimentos, en su opinión de 29 de enero de $2002^{16}$, indica entre otras recomendaciones que «es esencial tomar precauciones para reducir al mínimo los riesgos de reacciones alérgicas a productos $\mathrm{MG}$ » y que «son necesarios estudios de toxicidad sobre animales de laboratorio para evaluar los efectos de una exposición prolongada a pequeñas dosis de organismos modificados genéticamente sobre órganos vitales, en particular los sistemas inmunitario, hormonal y reproductor». Por su parte, en un informe publicado en febrero de $2002^{17}$ la Royal Society británica recomienda una mayor atención a los ingredientes modificados genéticamente en los productos para niños pequeños y a los posibles fenómenos alérgicos por inhalación. Sugiere un seguimiento después de la puesta en el mercado de los productos modificados genéticamente, en particular para vigilar las eventuales apariciones de alergias en grupos de riesgo como los niños. Todos estos aspectos no se contemplan en los procesos de aprobaciones de organismos modificados genéticamente que se están realizando en la actualidad. En cuanto a la Agencia Federal de Medio Ambiente de Austria, declaró en un monográfico de $2002^{18}$ que en los expedientes presentados para la autorización de organismos modificados genéticamente en la Unión Europea, «las investigaciones experimentales toxicológicas sólo se han llevado a cabo esporádicamente [...] En ninguno de los casos los efectos toxicológicos potencialmente relevantes de la inserción de los genes han sido considerados [...] En ninguno de los expedientes figuraba el estudio directo mediante experimentación de las propiedades alergénicas potenciales de la planta modificada genéticamente y/o del producto derivado de ella». 


\section{¿Y SI PASARA ALGO?}

Tal como se ha dicho antes, los estudios científicos publicados hasta la fecha sobre los efectos que pudieran tener los alimentos modificados genéticamente sobre la salud son muy pocos, aunque hubiera sido conveniente que la sociedad desarrollase este tipo de investigaciones antes de aceptar la introducción de estos alimentos en la cadena alimentaria.

El Dr. Putzai, en un libro publicado en $2003^{19}$, hace una revisión exhaustiva de los estudios llevados a cabo sobre el tema y concluye que los datos disponibles son insuficientes y de baja calidad para atreverse a afirmar que los alimentos modificados genéticamente son inocuos y saludables. A parte de las incógnitas sobre los impactos potenciales directos para la salud humana de este tipo de alimentos faltan aun más datos (si cabe) sobre los eventuales efectos de productos procedentes de animales que hayan sido alimentados con organismos modificados genéticamente. Aunque menos pesimista, la British Medical Association (BMA) llega a la misma conclusión: en su Segunda Declaración sobre los Alimentos Modificados Genéticamente y la Salud de marzo de $2004^{20}$, afirma: a nuestro juicio, el potencial de los alimentos modificados genéticamente de causar efectos perjudiciales sobre la salud es muy pequeño y muchos de los temores expresados se aplican igualmente a los alimentos convencionales. Sin embargo, las preocupaciones en relación con la seguridad no se pueden descartar plenamente hoy por hoy sobre la base de la información disponible actualmente. La BMA ha identificado varias áreas donde se tiene que llevar a cabo más investigación. Algunas de ellas son: la aparición de nuevas alergias, las características nutricionales (en particular para grupos vulnerables como el feto, los niños o las mujeres embarazadas) y la transferencia de genes dentro del aparato digestivo.

De la misma forma, en general los estudios científicos publicados sobre el tema invitan a más investigaciones para aportar luz sobre los efectos para la salud de los organismos modificados genéticamente. Por citar algunos ejemplos: un equipo de científicos holandeses ${ }^{21}$ ha encontrado que secuencias de aminoácidos presentes en proteínas transgénicas son idénticas a secuencias en proteínas alergénicas; en experimentos con ratones se ha encontrado que la respuesta inmunológica a la proteína Cry $1 \mathrm{Ab}$ de algunas plantas Bt es elevada y que algunas células involucradas en el metabolismo de asimilación de los alimentos ven sus características afectadas por la soja modificada genéticamente ${ }^{22,23}$. Todos concluyen que es necesario investigar más antes de introducir el tipo de organismo modificado genéticamente estudiado en concreto o los transgénicos en general en la cadena alimentaria.

En otro plano, la introducción de los cultivos modificados genéticamente tal y como los conocemos en la actualidad tiene como consecuencia el aumento del uso de herbicidas, especialmente el glifosato y el glufosinato de amonio. Aunque las empresas que comercializan estos productos (Monsanto y Bayer respectivamente) proclaman que tienen una toxicidad reducida, investigaciones independientes demuestran que esta afirmación no es tan cierta. Las preguntas sobre los posibles impactos negativos para la salud humana y el medio ambiente deben resolverse antes de que los cultivos tolerantes a estos productos obtengan los permisos para su liberación al medio ambiente. Dos revisiones de la literatura existente a este respecto hacen temer problemas para la salud como envenenamiento, eccema, dificultades respiratorias, tensión alta y efectos sobre el sistema reproductor en caso de exposición al glifosato y efectos sobre el sistema nervio en el caso del glufosinato de amonio ${ }^{24,25}$.

En Norte América, donde los cultivos y alimentos transgénicos están ya muy presentes, algunos científicos empiezan a pedir un mayor análisis de los organismos modifica- 
dos genéticamente en relación con sus posibles efectos sobre la salud. Es el caso del Comité de Identificación y Asesoramiento sobre los Efectos No Deseados de los Alimentos Modificados Genéticamente en los Humanos $^{26}$ creado por la Academia Nacional de Ciencias que en su informe de julio de 2004 hace siete recomendaciones para mejorar la evaluación de riesgo previa a la comercialización y el seguimiento una vez introducido en el mercado. Por su parte, en un informe de enero de $2003^{27}$, Doug Gurian-Sherman, identifica fallos importantes en los procedimientos de autorización de los organismos modificados genéticamente por la Food and Drug Agency (FDA), después de haber revisado los expedientes de aprobación. Entre otras medidas recomienda a la FDA desarrollar un protocolo detallado para determinar la seguridad y unas pautas de experimentación, así como realizar un nuevo examen de los cultivos modificados genéticamente ya comercializados en caso de que aparezcan dudas o datos novedosos. También un panel de expertos de la Royal Society of Canada (RSC), la academia canadiense de las ciencias y humanidades, declaró en 2001 que si fuera por ellos, los cultivos $y$ alimentos estarian sometidos a pruebas más rigurosas y los estudios estarían revisados por expertos independientes ${ }^{28}$.

Los organismos modificados genéticamente entran en nuestros alimentos desde hace varios años, en pequeña cantidad, pero regularmente. Hay que reconocer que en España no se ha detectado ningún caso grave de efecto sobre la salud. Sin embargo, en todos estos años, el sistema de etiquetado vigente no facilitaba una información completa. Desde abril de 2004 la legislación a este respeto ha cambiado, aportando algunas mejoras. Pero sigue siendo muy deficiente (a parte de que no se cumple) si se quiere hacer un seguimiento de los problemas sanitarios potenciales asociados a un consumo reducido pero diario de organismos modificados genéticamente. Los ingredientes transgénicos pasan prácticamente desapercibidos y sin embargo entran en nuestra dieta cotidiana. No existe en la actualidad ninguna posibilidad de establecer una relación causa/efecto entre el tipo de consumo que hacemos de los transgénicos y la aparición de nuevas patologías, como por ejemplo las alergias. La falta absoluta de certidumbres científicas sobre la seguridad de los alimentos transgénicos entra en contradicción con la falta de mecanismos que permitan detectar problemas eventuales y sobre todo con la rapidez con la que se han introducido estos nuevos alimentos en la cadena alimentaria.

\section{LA CONTAMINACIÓN POR ORGANISMOS MODIFICADOS GENÉTICAMENTE: UN FACTOR DE RIESGO AÑADIDO}

Los cultivos modificados genéticamente plantean serios problemas ambientales y para la agricultura no transgénica, que han ido apareciendo poco a poco a lo largo de esta primera década de liberación al medio ambiente. Una de las más graves es la contaminación genética, es decir la aparición de material modificado genéticamente en cultivos y productos no transgénicos, por polinización cruzada, esparcimiento de semillas, mezcla de las semillas y cosechas, etcétera.

Uno de los casos más emblemáticos de contaminación por organismos modificados genéticamente ha sido el caso StarLink, variedad de máiz transgénico autorizada en Estados Unidos como alimento para animales solamente. No obtuvo la autorización para el consumo humano debido al potencial alergénico de la proteína Cry9C presente en este maíz. En el año 2000 se detectó la presencia de StarLink en la cadena alimentaria humana. La magnitud y gravedad de la contaminación con StarLink fue pasmosa. Más de 300 productos alimenticios derivados del maíz fueron retirados del mercado en todo el país. A pesar del hecho que el StarLink sólo representaba el $0,4 \%$ del total de la superficie de maíz plantado la cantidad de hectáreas 
contaminadas fue mucho mayor. Más sorprendente aún fue que la proteína Cry9C, que supuestamente debía estar presente solamente en las semillas de maíz (amarillo) StarLink, se encontró en otras ochenta variedades de semillas de maíz amarillo, y más inesperadamente todavía en un producto derivado del maíz blanco.

La experiencia estadounidense con transgénicos ofrece otro ejemplo más del gran riesgo para el medio ambiente y la salud humana: 'los biofármacos'. La biofarmacología es una aplicación experimental de la biotecnología en la cual se manipulan genéticamente las plantas para que produzcan proteínas y sustancias químicas farmacéuticas. Entre los ejemplos conocidos se cuentan un anticonceptivo, poderosas hormonas de crecimiento, un coagulante sanguíneo, anticoagulantes sanguíneos, enzimas industriales y vacunas. En noviembre de 2002 se registró el primer caso importante de contaminación por biofármacos cuando ProdiGene, la empresa implicada, realizaba pruebas de campo con este tipo de cultivos y no retiró todos los remanentes del maíz transgénico biofármaco. Algunas semillas quedaron en el campo, geminaron y contaminaron el cultivo del año siguiente (soja). Más adelante el Departamento de Agricultura ordenó la destrucción de unas 500 mil toneladas de soja.

Según un estudio publicado recientemente por la Unión de Científicos Preocupa$\operatorname{dos}^{29}$, los cultivos transgénicos han provocado en pocos años una alarmante contaminación de las semillas convencionales, así como de algunos bancos de germoplasma fundamentales para la mejora genética de variedades. Los investigadores encontraron que más del $50 \% / 80 \%$ respectivamente, de las semillas convencionales de maíz/soja y colza contenían material modificado genéticamente.

Tenemos que aprender de las experiencias. Es evidente que la introducción a gran escala de cultivos transgénicos en el campo significa la desaparición a medio plazo de las semillas y productos libres de transgénicos. Sin embargo, dado el tremendo nivel de incertidumbre sobre la seguridad de los organismos modificados genéticamente resulta esencial preservar una agricultura libre de transgénicos, no sólo para garantizar el derecho a la libre elección del consumidor, sino para poder dar marcha atrás en caso de detectarse un problema. De lo contrario se hipotecaría el futuro de la agricultura lo que supone otro factor importante a medio/largo plazo de inseguridad alimentaria mundial.

\section{Futuro para la agricultura con los cultivos transgénicos}

El 81\% de las plantas modificadas genéticamente cultivadas en el mundo actualmente incorporan una tolerancia a un herbicida químico. La experiencia de Estados Unidos está demostrando que el uso de estos productos de síntesis aumenta con la introducción de las semillas transgénicas. Por ejemplo este aumento ha sido de un 5\% en el caso de la soja modificada genéticamente comparado con su equivalente convencional y hay indicios para pensar que el aumento es mucho mayor en Argentina ${ }^{30}$. Así mismo un reciente estudio del Northwest Science and Environmental Policy Center demuestra que la siembra de 220 millones de hectáreas de maíz, soja y algodón transgénicos desde 1996 ha tenido como consecuencia un aumento del uso de los herbicidas en torno a los 22 millones de $\mathrm{kg}^{31}$. Este hecho tiene consecuencias evidentes para el medio ambiente, tanto de contaminación de suelos, acuíferos, etcétera, como de la destrucción de la biodiversidad.

En cuanto a la proteina Bt, el $26 \%$ de los cultivos modificados genéticamente empleados la incorporan. Son muchas las investigaciones llevadas a cabo acerca de los efectos de la toxina $\mathrm{Bt}$ de las plantas transgénicas 
(que no tiene las mismas propiedades que la toxina en su forma natural producida por la bacteria Bacillus thuringiensis) sobre las especies no objetivo. Efectos negativos sobre mariposas ${ }^{32-34}$, insectos no voladores $^{35}$, crisopas $^{36,37}$ o coleópteros ${ }^{38}$ han sido reportados así como posibles impactos en animales insectívoros, como pájaros, murciélagos u otros componentes de la cadena trófica ${ }^{39}$. Por otra parte, se ha verificado que la toxina Bt puede permanecer activa en el suelo hasta 234 días $^{40-42}$. A través de las raíces de las planta, el polen o los restos de cosecha, la presencia de la toxina puede llegar a ser suficientemente alta como para poner en peligro organismos, insectos beneficiosos y otros tipos de animales como las lombrices ${ }^{43-45}$.

Otras de las preocupaciones relacionadas con las plantas modificadas genéticamente es la aparición de resistencias a la toxina $\mathrm{Bt}$ en las poblaciones de insectos ${ }^{46,47}$. Una exposición continua a la toxina producida por la planta fomenta la supervivencia de los individuos que presentan una inmunidad genética al Bt. La probabilidad de aparición de resistencias es mucho mayor con los cultivos Bt que con la toxina procedente de la bacteria Bacillus thuringiensis en su forma natural porque esta última se degrada rápidamente bajo la influencia de la luz del día. En cambio, la producción continua de la toxina por las plantas $\mathrm{MG}$, su alta concentración y su acumulación a lo largo del tiempo exponen continuamente las poblaciones al Bt. Este fenómeno crea plagas cada vez más fuertes que habrá que combatir con más productos químicos o con otras soluciones tecnológicas que las empresas del sector agroquímico se alegrarán vender.

Estos tres puntos ilustran qué futuro nos espera con la introducción de los organismos modificados genéticamente en la agricultura. Una agricultura cada vez más peligrosa para el medio ambiente y que requiere soluciones cada vez más tecnológicas, cada vez más alejadas de los procesos naturales, como continuación del modelo industrial de producción agrícola que se ha ido imponiendo en las últimas décadas. Este modelo implica impactos importantes como la desaparición de la biodiversidad agrícola, la contaminación del suelo, de las aguas, de la atmósfera, etc., repercutiendo todo ello sobre la seguridad alimentaria, aunque sea de forma indirecta.

\section{Una agricultura hecha a la medida de cinco multinacionales}

El asunto más preocupante (si cabe) de la introducción de los organismos modificados genéticamente en la agricultura es el monopolio que cinco grandes empresas transnacionales están creando sobre el mercado de semillas y productos agroquímicos. Esta tendencia, ya real sin los transgénicos, se ve exacerbada por la aparición de las variedades modificadas genéticamente, propiedad de la empresa (en algunos países hasta limites insospechables) y en general compatible con un producto químico determinado. Con ello la dependencia del agricultor hacía la empresa es total. El dominio de la tolerancia a un herbicida con las características de las variedades transgénicas actualmente en el mercado no es de extrañar: esta propiedad sirve para incrementar la venta del herbicida asociado a la semilla.

La creciente concentración de las grandes empresas transnacionales que empezó en los años 90 ha afectado de lleno a los sectores de la agroalimentación/agroquímica y de la farmacia, creando unas grandes multinacionales con un inquietante poder para controlar los mercados agrícolas, sanitarios y alimentario $^{48}$. En lo que se refiere al mercado de las semillas transgénicas las ventas están monopolizadas por las cinco mayores compañías agroquímicas del mundo: Syngenta, Bayer CropScience, Monsanto, DuPont y Dow (todas con economías mayores a muchos países del tercer mundo ${ }^{49,50}$ ). Monsanto es la empresa que más semillas transgénicas ven- 
de: en su informe de 2003 figura que sus variedades cubren más del 90\% de la superficie total sembrada con cultivos transgénicos [en el mundo]» ${ }^{51}$. Estos datos deben invitar a una reflexión. Las tendencias de estos últimos años, reforzadas con la aparición de los cultivos transgénicos, hacen que los agricultores dependan cada vez más de un pequeño número de grandes empresas (que además se van concentrando) con las repercusiones que esto pueda tener sobre pueblos e incluso países. La seguridad alimentaria en términos de disponibilidad de alimentos está en juego.

\section{CONCLUSIÓN}

La rápida expansión de los cultivos modificados genéticamente en algunas partes del mundo contrasta con el poco conocimiento que tenemos en la actualidad de su impacto socio-económico, para la salud y para el medio ambiente. Sobre los mecanismos biológicos permanecen muchas incógnitas y nadie es capaz de predecir todos los efectos de la inserción de un gen en un organismo extraño, sobre todo cuando este organismo se libera al medio ambiente. Se sabe poco más en cuanto a sus efectos sobre la salud humana y animal y son numerosas las voces científicas que opinan que los procedimientos de autorización de alimentos transgénicos en la Unión Europea son deficientes en cuanto al análisis de riesgo. A esto se añade que si un organismo modificado genéticamente resultase perjudicial para la salud, no lo podríamos detectar por falta de mecanismos adecuados, en particular por las deficiencias del sistema de etiquetado.

En segundo lugar, la introducción de los cultivos transgénicos en la agricultura se está realizando sin las medidas de precaución imprescindibles dado el nivel de incertidumbre que los rodea. Los impactos ambientales y sobre el resto de la agricultura, como la contaminación genética, la pérdida de biodiversidad agrícola y silvestre, la aparición de plagas más difíciles de controlar, la dependencia cada vez mayor hacia los agroquímicos, entre otros productos, se hacen cada día más evidentes. Impactos que repercuten indudablemente sobre la seguridad alimentaria e hipotecan el futuro de la agricultura.

Por último, estos cultivos intensifican la industrialización de la agricultura ya que se prioriza la producción de cultivos comerciales para el mercado mundial en detrimento de las necesidades de las comunidades locales y de la biodiversidad agrícola, clave para la soberanía alimentaria. A la vez, deja caer la agricultura y alimentación mundial en manos de un puñado de grandes empresas transnacionales. Por lo tanto no se puede afirmar que los cultivos modificados genéticamente contribuirán a un reparto más justo de los recursos naturales entre todos los seres humanos, a una disminución de las desigualdades entre países ricos y países pobres y a la resolución del problema del hambre en el mundo. Es de temer que, al contrario, este modelo de agricultura será uno de los factores que incrementen las diferencias entre pobres y ricos.

Todos estos elementos hacen que la introducción de los organismos modificados genéticamente atenten contra la sostenibilidad, ya que no aportan soluciones satisfactorias ni desde el punto de vista medioambiental, social ni económico ${ }^{52}$ y pone en peligro un modelo de agricultura que asegure la satisfacción de las necesidades de las generaciones presentes sin comprometer la capacidad de las generaciones futuras para satisfacer las suyas.

Existen alternativas viables y prácticas a los cultivos transgénicos que casi invariablemente resultan más baratas, más accesibles, más productivas en ambientes marginales y más aceptables social y culturalmente. La oposición ciudadana a los transgénicos y los enormes riesgos inherentes a su utilización deberían catalizar un cambio de enfoque 
hacia las técnicas agrarias alternativas y confiables, que son menos costosas que la multimillonaria industria moderna de la biotecnología.

\section{BIBLIOGRAFÍA}

1. James C. Preview: Global Status of Commercialized Transgenic Crops: 2003. ISAAA Briefs No. 30. New York: Ithaca; 2003.

2. Sentís C. Transgénicos, cara y cruz. El cultural (suplemento de El Mundo) 2002; 22 de mayo.

3. Gibbs WW. The unseen genome: gems among the junk. Sci Am. 2003 Nov;289(5):26-33. Erratum in: Sci Am. 2004 Mar;290(3):14

4. Lee TI, Rinaldi NJ, Robert F, Odom DT, BarJoseph Z, Gerber GK et al. Transcriptional regulatory networks in Saccharomyces cerevisiae. Science 2002;298:799-804.

5. A. Aguirre de Cárcer. Descubierto un misterioso fragmento de material genético en la soja de Monsanto. ABC 2001. 17 de agosto.

6. Friends of the Earth Europe. Questions remaining over Monsanto's NK603 maize. 02/2004. Disponible en: http://www.foeeurope.org/GMOs/pending/nk603briefing.pdf

7. Moens W. Report on the molecular characterization of the genetic map of event Bt11. Brussels; Scientific Institute of Public Health; 2003 Disponible en: http://www.biosafety.be/TP/MGC_reports/Report Bt11.pdf

8. Mae-Wan Ho. Unstable Transgenic Lines Illegal. Nota de Prensa de ISIS 03/12/03. Disponible en: www.i-sis.org.uk

9. Pryme IF, Lembcke R. In vivo studies on possible health consequences of genetically modified food and feed-with particular regard to ingredients consisting of genetically modified plant materials. Nutr Health. 2003;17(1):1-8.

10. Domingo Roig JL, Gómez Arnáiz M.. Riesgos sobre la salud de los alimentos modificados genéticamente: una revisión bibliográfica. Rev Esp Salud Pública 2002; 74: 225-61.

11. Friends of the Earth. Comentarios a varias aplicaciones de autorización en la Unión Europea de organismos modificados genéticamente: FoEE assessments of the pending GMO authorisations: Monsanto's GM Roundup Ready Oilseed rape GT73; Bayer's GM Oilseed rape MS8xRF3; Monsanto's GM Maize Mon 863 ; Syngenta's GM Maize Bt11 ; Monsanto's GM Maize NK603 ; Bayer's GM Rice LLRICE62 ; Mycogen/DowAgrosciences GM Cotton 281-24-236/3006-210-23. Disponible en: http://www.foeeurope.org/GMOs/ pending/index.htm

12. Agence Française de Sécurité Sanitaire des Aliments. Avis relatif aux compléments d'information concernant le dossier de mise sur le marché du «Maïs doux Bt 11 (pZO1502)» au titre du règlement 258/97/CE. París: Agence Française de Sécurité Sanitaire des aliments; 2003. Disponible en: http://www.afssa.fr/ftp/afssa/23721-23722.pdf

13. Moreno W. Report on the molecular characterization of the genetic map of event Bt 11. Brussel: Scientific Institute of Public health; 2003. Disponible en biosafety.be/TP/MbC_report_Bt11.pdf

14. Gaugitsch H, Spök A, Hofer H, Lehner P, KienzlPlochberger K, Valenta R. Toxikologie und Allergologie von GVO-Produkten. Roten Reihe des Bundesministeriums für Gesundheit und Frauen Sektion IV, Band /03. 2003.

15. European Food Safety Authority. EFSA provides scientific advice on the use of antibiotic resistance marker genes in genetically modified plant. Nota de Prensa del 19/04/2004. Disponible en: http://www.efsa.eu.int/press_room/press_release/3 86/press_release_0604_gmo_en1.pdf

16. Agence Française de Sécurité Sanitaire des aliments. Evaluation des risques relatifs à la consommation de produits alimentaires composés ou issus d'organismes génétiquement modifiés - París: Agence Française de Sécurité Sanitaire des aliments; 2002. Disponible en: http://www.afssa.fr/ ftp/basedoc/1999sa0035.pdf

17. The Royal Society (UK). Genetically modified plants for food use and human health; an update. London: The Royal Society (UK); 2002.

18. Spök A., Hofer H., Valenta R., Kienzl-Plochberger K., Lehner P., Gaugitsch H. Toxikologie und Allergologie von GVO-Produkten. Monographien Band 109, UBA. Viena: Federal Environment Agency; 2002 .

19. A Pusztai, S. Bardocz, S.W.B. Ewen. Genetically modified food: potential human health effects en: Food Safety: contaminants and toxins. D'Mello JPF editor. Edinburgh: Scottish Agricultural College;2003. 
20. British Medical Association. Board of Science and Education. Genetically modified foods and health: a second interim statement. London: British Medical Association;2004.

21. Kleter GA, Peijnenburg AA. Screening of transgenic proteins expressed in transgenic food crops for the presence of short amino acid sequences identical to potential, IgE - binding linear epitopes of allergens. BMC Struct Biol 2002 Dec 12;2(1):8.

22. Vazquez-Padron RI, Moreno-Fierros L, NeriBazan L, Martinez-Gil AF, de-la-Riva GA, LopezRevilla R. Characterization of the mucosal and systemic immune response induced by Cry1 Ac protein from Bacillus thuringiensis HD 73 in mice. Braz J Med Biol Res 2000;33(2):147-55.

23. Malatesta M, Caporaloni C, Gavaudan S, Rocchi MBL, Serafini S, Tiberi C and Gazzanelli G. Ultrastructural Morphometrical and Immunocytochemical Analyses of Hepatocyte Nuclei from Mice Fed on Genetically Modified Soybean. Cell Struct. Funct 2002; 27:173-80

24. Buffin D and Jewell T. Health and environmental impacts of glyphosate: The implications of increased use of glyphosate in association with genetically modified crops.07/2001. Riley P, Taylor $\mathrm{M}$, Diamand $\mathrm{E}$ and Barron $\mathrm{H}$ editors. London: Friends of the earth; 2001. Disponible en: http:/www.foe.co.uk/resource/reports/impacts gl yphosate.pdf

25. Jewell Tand Buffin D. Health and environmental impacts of glufosinate ammonium. Riley P, Taylor M, Diamand E and Barron $\mathrm{H}$ editors. London: Friends of the earth; 2001. Disponible en: http:/www.foe.co.uk/resource/reports/impacts gl ufosinate_ammon.pdf

26. Masters BS et all. Safety of Genetically Engineered Foods: Approaches to Assessing Unintended Health Effects. Committee on Identifying and Assessing Unintended Effects of Genetically Engineered Foods on Human Health, National Research Council; 2004. Disponible en: http://books.nap. edu/execsumm_pdf/10977.pdf

27. Doug Gurian-Sherman. Holes in the Biotech Safety Net: FDA Policy Does Not Assure the Safety of Genetically Engineered Foods. Center for Science in the Public Interest.;2003. Disponible en: http://www.cspinet.org/new/pdf/fda report final. pdf

28. The Royal Society of Canada. Expert panel raises serious questions about the regulation of GM food. Ottawa: The Royal Society of Canadá; 2001. Dis- ponible en: http://www.rsc.ca/foodbiotechno$\operatorname{logy} /$ GMstatementEN.pdf

29. Mellon M, Rissler J. Gone to Seed. Tansgenic Contaminants in the Traditional Seed Supply. Union of Concerned Scientists;2004

30. Charles M. Benbrook. GMOs, Pesticide Use, and Alternatives. Lessons from the U.S. Experience. Presented at the Conference on GMOs and Agriculture - Paris France; 20/06/2003 Disponible en: http://www.biotech-info.net/lessons learned.html

31. Benbrook cm. Impacts of Genetically Engineered Crops on Pesticide Use in the United States: The First Eight Years. Idaho: Northwest Science and Environmental Policy Center; 2003. Disponible en: http://www.biotech-info.net/technicalpaper6.html

32. Hanson-Jesse LC, Obrycki JJ. Field deposition of Bt transgenic corn pollen: Lethal effects on the monarch butterfly. Oecologia 2000;125:.241-8.

33. 1: Hellmich RL, Siegfried BD, Sears MK, StanleyHorn DE, Daniels MJ, Mattila HR, Spencer T, Bidne $\mathrm{KG}$, Lewis LC. Monarch larvae sensitivity to Bacillus thuringiensis- purified proteins and pollen.Proc Natl Acad Sci U S A 2001;98(21): 11925-30

34. Zangerl AR, McKenna D, Wraight CL, Carroll M, Ficarello P, Warner R, Berenbaum MR. Effects of exposure to event 176 Bacillus thuringiensis corn pollen on monarch and black swallowtail caterpillars under field conditions.Proc Natl Acad Sci U S A. 2001;98(21):11908-12. .

35. Bt maize (corn) leaf protein (LP176-0194) - 28 day survival and reproduction study in Collembola (Folsomia candidia) EPA MRID No 434635-01.

36. Hilbeck A, Baumgartner M, Fried PM, Bigler F. Effects of transgenic Bacillus thuringiensis cornfed prey on mortality and development time of immature Chrysoperla carnea (Neuroptera: Chrysopidae). Environ Entomol 1998; 27: 480-7.

37. Hilbeck A, Moar WJ, Pusztai-Carey M, Filippini A, Bigler F. Prey-mediated effects of Cry1 Ab toxin and protoxin and Cry2A protoxin on the predator Chrysoperla carnea. Entomol Exp Appl 1999; 91: 305-16.

38. Wold SJ, Burkness EC, Hutchison WD, Venette $\mathrm{RC}$. In-field monitoring of beneficial insect populations in transgenic sweet corn expressing a Bacillus thuringiensis toxin. J Entomol Sci 2001; 36: 177. 
39. Kenneth RF. Genetic Engineering of Trees to Enhance Resistance to Insects. BioScience 1989; 39:532-3.

40. USA Environmental Protection Agency. Report from the FIFRA Scientific Advisory Panel meeting on Bt plant-pesticides risk and benefit assessments, October 18-20,2001. Disponible en: www.epa.gov/scipoly/sap/2000/index.htm\#october

41. Koskella J, Stotzky G. Microbial utilization of free and clay-bound insecticidal toxins from $\mathrm{Bt}$ and their retention of insecticidal activity after incubation with microbes. Applied and Env. Microbiology 1997; 3561-8.

42. Tapp H., Stotzky G. Persistence of the insecticidal toxin from Bt subsp. Kurstaki in soil. Soil Biology and Biochemistry 1998;30: 471-6.

43. Sacena D, Flores S, Stotzky G. Transgenic plants: Insecticidal toxin in root exudates from Bt corn. Nature 1999:402-0.

44. Zwahlen C, Hilbeck A, Gugerli P, Nentwig W. Degradation of the Cry1 Ab protein within transgenic Bt corn tissue in the field. Mol Ecol 2003;12:.765-75

45. Zwahlen, C., Hilbeck, A., Howald, R. and Nentwig, W. Effects of transgenic Bt corn litter on the earthworm Lumbricus terrestris. Mol Ecol2003; $12: 1077-86$

46. Androw DA. Resisting Resistance to Bt corn. En Letourneau DK and Burrows BE. Genetically engineered organisms: assessing environmental and human health effects. Boca Raton, FL: CRC Press.

47. Amigos de la Tierra y Greenpeace. Al grano: impacto del maíz transgénico en España. Madrid: Amigos de la Tierra y Greenpeace; 2003.

48. Riechmann J. Qué son los alimentos transgénicos. Madrid:Integral;2002.

49. Corporate Watch. GM Crops Industry Overview. The Big Three Prepare To Commercialise Oxford: Corporate watch biotech briefings; 2003. Disponible en: http://www.corporatewatch. org.uk/genetics/commercialisation/overview_final.pdf

50. United Nations Development Programme. Estadísticas sobre desarrollo humano. Disponible en: http:/hdr.undp.org/statistics/data/

51. Monsanto Company - A clear Focus. Annual Report 2003. Monsanto; 2003.

52. Soil Association. Seeds of doubt: North American farmers' experiences of GM crops. Bristol: Soil Association; 2002. Disponible en: www.soilassociation.org 